\title{
Nonlinear Refraction and Absorption \\ of Ag29 Nanoclusters : Evidence for Two-Photon Absorption Saturation
}

\author{
Albert S. Reyna, ${ }_{1}$ Isabelle Russier-Antoine, ${ }^{2}$ Franck Bertorelle, ${ }^{2}$ Emmanuel Benichou, ${ }^{2}$ \\ Philippe Dugourd, ${ }^{2}$ Rodolphe Antoine, ${ }^{2}$ Pierre-François Brevet, ${ }^{2 *}$ Cid B. de Araújo ${ }^{1}$ \\ ${ }^{1}$ Departamento de Física, Universidade Federal de Pernambuco, 50670-901, Recife, PE, \\ Brazil \\ ${ }^{2}$ Institut Lumière Matière, UMR5306 Université Lyon 1-CNRS, Université de Lyon, 69622 \\ Villeurbanne Cedex, France
}

Corresponding author : Prof. Pierre-François Brevet, pfbrevet@,univ-lyon1.fr 


\begin{abstract}
We report the nonlinear absorption and refraction of Ag29 nanoclusters aqueous suspensions using the open and closed aperture Z-scan method at $532 \mathrm{~nm}$. The stoichiometry of the atomically precise silver nanoclusters has been determined by mass spectrometry and their formula is $\mathrm{Ag}_{29} \mathrm{DHLA}_{12}$ where the ligand is dihydrolipoic acid (DHLA). Using different concentration of nanoclusters, we observe beyond the expected linear absorption saturation and nonlinear absorption, a clear saturation of the nonlinear absorption, a feature so far not described for nanoclusters. The nonlinear refraction is also determined and exhibits a regular behavior with a negative $\mathrm{n}_{2}$ value. A figure of merit is discussed in view of potential applications.
\end{abstract}




\section{Introduction}

Interaction of intense light beams with matter can induce various nonlinear optical effects and phenomena. Among them, nonlinear absorption and refraction, two processes describing the change of the optical absorption and refraction index of a material with the incident light intensity, have received considerable attention. ${ }^{1-3}$ This interest lies in the numerous applications offered in a wide range of fields, from spectroscopy, laser science and microscopy to functional material design. ${ }^{4-8}$ Recently, the search for materials with large nonlinear absorption or nonlinear refraction has turned towards nanomaterials and heterogeneous materials with embedded nanoscale structures. ${ }^{9-12}$ Matrices incorporating metallic nanoparticles have in particular been shown to possess extremely large nonlinear absorption or refraction due to the presence of confined conduction band electrons. Besides, for particles with diameters smaller than about $100 \mathrm{~nm}$ in particular, surface plasmon resonances (SPR), namely resonances associated with the collective excitation of these conduction band electrons, provide further enhancements of these nonlinear optical properties. Within the visible optical spectrum, gold and silver are the most commonly studied metals although copper and platinum have also been characterized. ${ }^{13,14}$ Metallic nanoparticles however only supports SPR when they possess sufficiently large diameters and therefore a sufficiently large number of atoms. For instance, gold nanoparticles with a number of atoms below about 150 do not support anymore SPR resonances and rather behave like large molecules. ${ }^{15}$ Nevertheless, these ultrasmall nanoparticles, also known as nanoclusters and constituted from only a few metal atoms stabilized by organic ligands, still exhibit large nonlinear optical properties that are currently at the focus of an intense research effort worldwide. $^{16-18}$

The nonlinear optical properties of gold nanoclusters, and in particular the two-photon absorption cross-section (TPA), are interesting to develop high resolution multiphoton excited 
fluorescence imaging or optical limiting applications. ${ }^{19}$ Two-photon absorption, i.e. 2PA or TPA, is a nonlinear absorption phenomenon involving a direct transition from a low-lying state, in general the ground state, to high-lying states by the simultaneous absorption of two photons. Two successive one-photon excitations can be difficult to disentangle from direct TPA but the net result is rather similar. In the high intensity regime, TPA transitions can exhibit saturation. The simultaneous absorption of two photons from the exciting light promotes a great number of electrons into a finite number of high-lying states. The low-lying state thus becomes depleted and the absorption cannot further increase with intensity. As opposed to saturated absorption (SA), the phenomenon of reversed saturated absorption (RSA) including excited state absorption (ESA) may counteract saturation as incident photons may be absorbed by excited states when their population gets large enough. From this brief overview, it is rather obvious that not only the incident photon intensity but also the incident frequency are critical and may lead to different behaviors depending on the resonant or non resonant character of the transitions involved.

For nanoparticles, the transition from the molecular to plasmonic regime for nonlinear absorption has been investigated for gold and silver metals. ${ }^{15,20}$ For gold nanoclusters with an atom number below about 150, a non saturated TPA is observed at $532 \mathrm{~nm}$ excitation wavelength. Indeed, the saturation intensity of the nonlinear absorption is expected to be far too large in most cases, typically much beyond $10^{8} \mathrm{~W} / \mathrm{cm}^{2}$. For plasmonic nanoparticles, while a strong TPA is observed, clear saturation of the linear absorption has been demonstrated as well as RSA. Hence, the nonlinear absorption coefficient $\alpha(I)$ where $I$ is the incident intensity can be described with the following general expression:

$$
\alpha(I)=\frac{\alpha_{0}}{1+\left(I / I_{S}\right)}+\alpha_{2} I
$$


where $\alpha_{0}$ is the linear absorption, $I_{S}$ the saturation intensity and $\alpha_{2}$ the effective TPA coefficient incorporating both the direct TPA and successive one-photon absorption processes. For instance, for $\mathrm{Au}_{144}$ nanoclusters, expected to stand at the frontier between the molecular to the plasmonic transition, $I_{S}=1.5 \times 10^{8} \mathrm{~W} / \mathrm{cm}^{2}$ and $\alpha_{2}=7.5 \times 10^{-10} \mathrm{~m} / \mathrm{W}$ at $532 \mathrm{~nm}$ excitation wavelength. TPA can be described with the nonlinear absorption coefficient $\alpha_{2}$ with units of $\mathrm{m} / \mathrm{W}$ or the nonlinear absorption cross-section $\sigma_{2}$ with units of Göppert-Mayer $\left(\mathrm{GM}, 1 \mathrm{GM}=10^{-50} \mathrm{~cm}^{4} \mathrm{~s}\right) .{ }^{19}$ For the $\mathrm{Au}_{25} \mathrm{Capt}_{18}$ nanoclusters, where the ligand Capt stands for Captopril, $\sigma_{2}$ reaches $2.4 \times 10^{4} \mathrm{GM}$ at $830 \mathrm{~nm}$ excitation. ${ }^{21}$ The TPA cross-section for $\mathrm{Au}_{15} \mathrm{SG}_{13}$ is $6.5 \times 10^{4} \mathrm{GM}$ at $800 \mathrm{~nm}$ whereas it can reach values beyond $10^{6} \mathrm{GM}$ for plasmonic nanoparticles with a thousand atoms. ${ }^{15,17}$ Data are scarcer for silver nanoclusters. ${ }^{22}$ The TPA cross-sections at $800 \mathrm{~nm}$ reaches $10^{4} \mathrm{GM}$ for $\mathrm{Ag}_{29}(\mathrm{DHLA})_{12}$ where DHLA stands for dihydrolipoic acid as the ligand..$^{23}$ This value is only slightly smaller than that of $\mathrm{Au}$ nanoclusters at this wavelength. Nevertheless, it appears that large nonlinear absorptions are obtained at long wavelengths, close to $800 \mathrm{~nm}$. For a number of about 100 silver atoms, nanoclusters exhibit TPA coefficient of $\alpha_{2}=5 \times 10^{-10} \mathrm{~m} / \mathrm{W}$ and linear absorption saturation intensities in excess of $10^{8} \mathrm{~W} / \mathrm{cm}^{2}$ at $480 \mathrm{~nm} \cdot{ }^{20}$ All these results therefore suggest that Eq.(1) is valid for silver and gold nanoclusters and nanoparticles. However, experiments usually performed at high nanoclusters concentrations are strongly dominated by linear absorption. As a result, it is not possible to fully assess the range of validity of this expression, with respect to both intensity and concentration.

In many instances, experiments were performed for both open (OA) and closed (CA) aperture Z-scan configurations. Hence, it is possible to find data for nonlinear refraction as well. The general intensity dependent expression $n(I)$ of the refraction index writes as the sum of the linear intensity independent refraction index $n_{0}$ and the nonlinear counterpart $n_{2}$ as : 


$$
n(I)=n_{0}+n_{2} I
$$

where $n_{2}$ can be reported in units of $\mathrm{cm}^{2} / \mathrm{W}$ or GM, similarly to the TPA cross-section. ${ }^{24}$ For gold nanoclusters, $n_{2}$ is positive at wavelengths longer than $550 \mathrm{~nm}$.

In this work, we present a closed and open aperture Z-scan study to determine the nonlinear refraction and absorption of aqueous suspensions of $\mathrm{Ag}_{29}$ DHLA nanoclusters. In particular, for nonlinear absorption, we focus on the saturation phenomenon, previously unobserved for 2PA with nanoclusters. Three concentrations of the nanoclusters aqueous solutions are used, lending possible a full study of the sample behavior as a function of intensity and concentration over wide ranges. Likewise, linear refraction is investigated over the same range of intensities and concentrations in order to assess the suitability of nanoclusters for nonlinear optical applications.

\section{Materials and Methods}

Synthesis : The silver nanoclusters synthesis is based on the method already published by Adhikari et al.. ${ }^{25}$ The solution of the as-prepared silver nanoclusters has an orange color. The size of the prepared nanoclusters was assigned using Electrospray Ionization (ESI)-mass spectrometry, see Figure 1. 


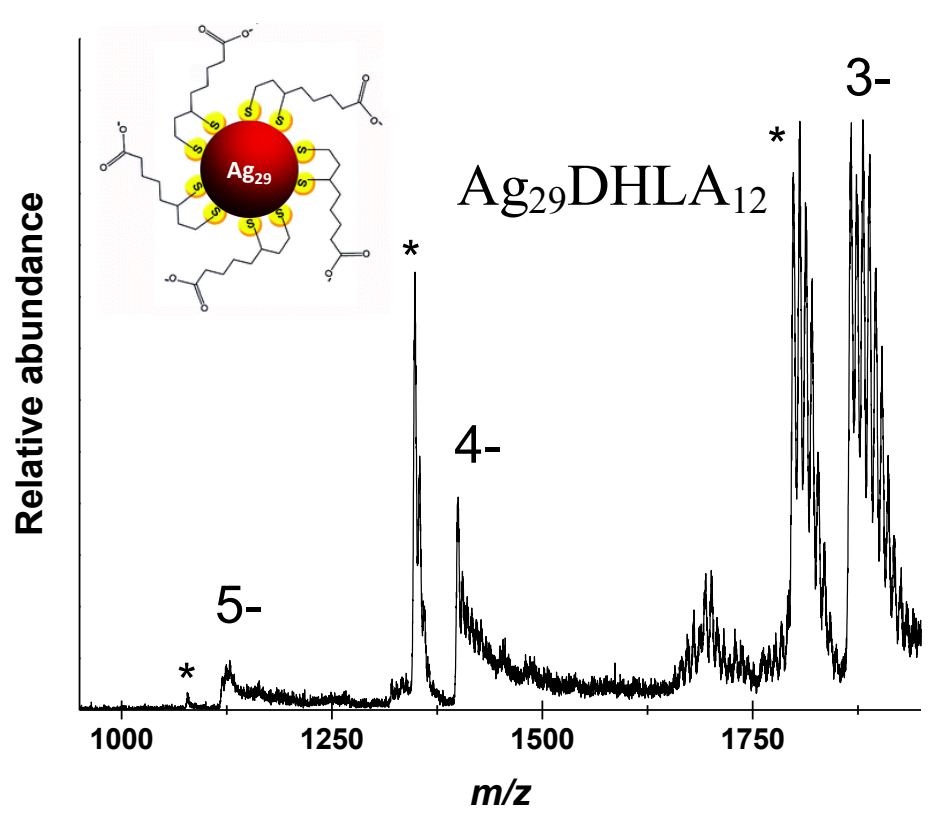

Figure 1: $\quad$ ESI mass spectrum of the synthesized $\operatorname{Ag}_{29}(\mathrm{DHLA})_{12}$ clusters. The asterisk corresponds to peaks assigned to the loss of one DHLA molecule from the Ag clusters (see text for details). Inset : schematics of the $\mathrm{Ag}_{29}$ nanocluster capped by dihydrolipoic acid (DHLA) ligands. For the sake of clarity, only 5 DHLA ligands are represented (among the 12 total ligands).

Three groups of broad ion signals, around $\mathrm{m} / \mathrm{z}=1130,1400$ and 1867 originate from the same nanoclusters in the respective $\mathrm{z}=5^{-}, 4^{-}$and $3^{-}$charge state. We previously identified these nanoclusters as $\mathrm{Ag}_{29} \mathrm{DHLA}_{12}$ on the basis of the good agreement of the theoretical and observed mass of the base peak in the deconvoluted spectrum. Deprotonation of the carboxylic acid group and subsequent association with $\mathrm{Na}^{+}$, termed $\mathrm{H}^{+} / \mathrm{Na}^{+}$exchange, results in the observation of multiple ion signals for the same clusters with the same overall charge with the general $\left[\mathrm{Ag}_{29} \mathrm{DHLA}_{12}-\mathrm{mH}^{+} \mathrm{pNa}^{+}\right]^{\mathrm{n}-}$ formula. 
The stoichiometry observed here is different from the one reported for gluthationeprotected gold nanoclusters and may be attributed to the fact the DHLA possesses two sulphur atoms leading to different ligand staple motifs. The colloidal solutions were prepared by addition of $0.15,1$ and $1.5 \mathrm{mg}$ of $\mathrm{Ag}$-nanoclusters powder into $1.5 \mathrm{~mL}$ of deionized water.

Linear optical properties : The linear optical properties of the DHLA protected silver nanoclusters were characterized by absorption spectroscopy in the UV-visible-NIR range, see Figure 2. The absorption spectrum reveals several bands with maxima at 328, 428 and $499 \mathrm{~nm}$ combined with a weak absorbance onset around $600 \mathrm{~nm}$. The linear optical properties reported here are similar to the ones previously reported. ${ }^{23,25,26}$

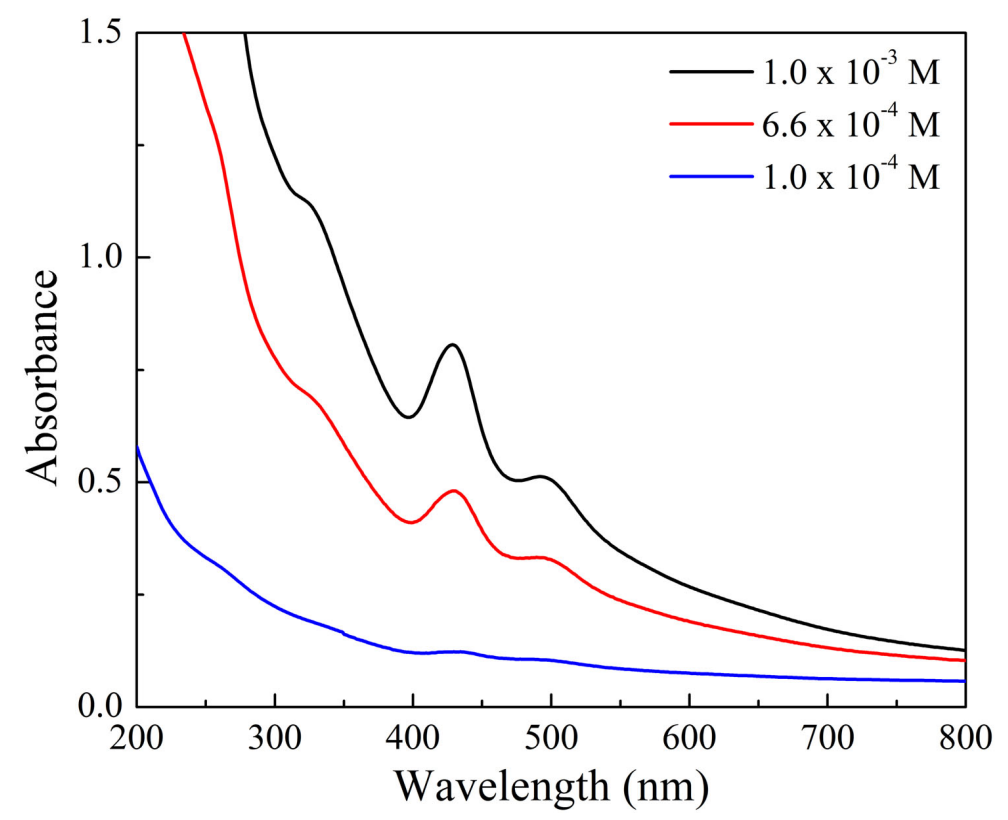

Figure 2: UV-visible NIR absorption spectra for the three concentrations of the silver nanoclusters aqueous solutions (Sample optical path length : $1 \mathrm{~mm}$ ). 
Nonlinear optical set-up : We used the second harmonic of a Q-switched and mode-locked Nd:YAG laser (pulse duration 80 ps, wavelength of $532 \mathrm{~nm}$, repetition rate of $10 \mathrm{~Hz}$ ). Single pulses were selected using a pulse picker to avoid the contribution of thermal effects. The incident pulses on the samples had maximum energy of $10 \mu \mathrm{J}$. Control of the total power and the linear polarization of the incident beam were accomplished with a $\lambda / 2$ plate followed by a Glan prism. For the Z-scan measurements, the Gaussian beam $\left(\mathrm{M}^{2}=1.1\right)$ was focused by a lens with a $10 \mathrm{~cm}$ focal length (beam waist of $20 \mu \mathrm{m}$ ) on a sample with thickness of $1 \mathrm{~mm}$, contained in a fused quartz cell. Slow photo-detectors placed in the far-field region with adjustable apertures in front of them were used to measure the beam intensity transmitted by the sample. The detected signals were processed by a boxcar integrator and a computer. Two schemes were used simultaneously, see Figure 3, namely closed (CA) and open (OA) aperture Z-scan, in order to determine the nonlinear refractive indices and the nonlinear absorption coefficients, respectively. A reference channel was used to improve the signal-to-noise ratio. Liquid carbon disulfide $\left(\mathrm{CS}_{2}\right)$ with nonlinear refractive index equal to $3.1 \times 10^{-14} \mathrm{~cm}^{2} / \mathrm{W}$ at 532 $\mathrm{nm}$ was used as reference standard for calibration measurements at low intensities. ${ }^{27}$ All data were checked against degradation by ensuring that the initial and final intensities collected as a function of the longitudinal displacement where identical.

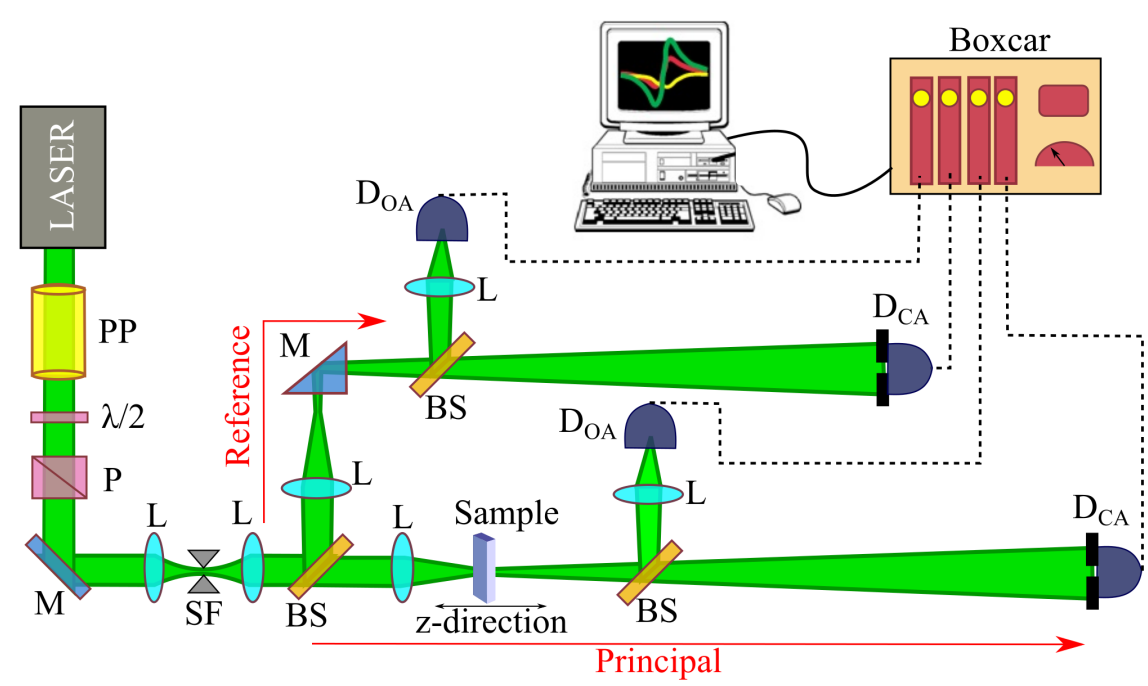


Figure 3 : $\quad \mathrm{OA}$ and CA Z-scan experimental setup. (PP) Pulse picker, (P) polarizer, (M) mirrors, (SF) spatial filter, (L) lenses, (BS) beam splitter and detectors for the closed-aperture $\left(\mathrm{D}_{\mathrm{CA}}\right)$ and open-aperture $\left(\mathrm{D}_{\mathrm{OA}}\right)$ schemes.

\section{Results and Discussion}

The normalized transmittance in the closed aperture CA Z-scan measurements as a function of the sample longitudinal displacement across the incident beam waist allows for the determination of the refractive part of the nonlinear optical index. This normalized transmittance was adjusted with the standard expression developed by Sheik-Bahae: ${ }^{28}$

$$
T(z)=1+\frac{4 \Delta \Phi_{0}^{(3)}\left(\mathrm{z} / \mathrm{z}_{0}\right)}{\left[\left(\mathrm{z} / \mathrm{z}_{0}\right)^{2}+9\right]\left[\left(\mathrm{z} / \mathrm{z}_{0}\right)^{2}+1\right]}
$$

with $\Delta \Phi_{0}^{(3)}=k n_{2} I\left[1-\exp \left(\alpha_{0} L\right)\right] / \alpha_{0}$ corresponding to the third-order phase-shift and $n_{2}$ the third-order refractive index. The wave number is $k=2 \pi / \lambda$ where $\lambda$ is the incident laser wavelength, $z_{0}$ is the laser Rayleigh length, $\alpha_{0}$ is the linear absorption coefficient, $I$ and $L$ the incident intensity and the sample length, respectively. The intensity dependent CA Z-scan plots for three different concentrations of the aqueous solution of the $\operatorname{Ag}_{29} \mathrm{DHLA}_{12}$ nanoclusters are shown below, Figure 4a-c. They all exhibit an increasing nonlinear refraction with incident intensity as well as a peak followed by a valley (displacement of the sample from before to after the focal plane of the laser), indicative of a negative $n_{2}$ value. 
(a)

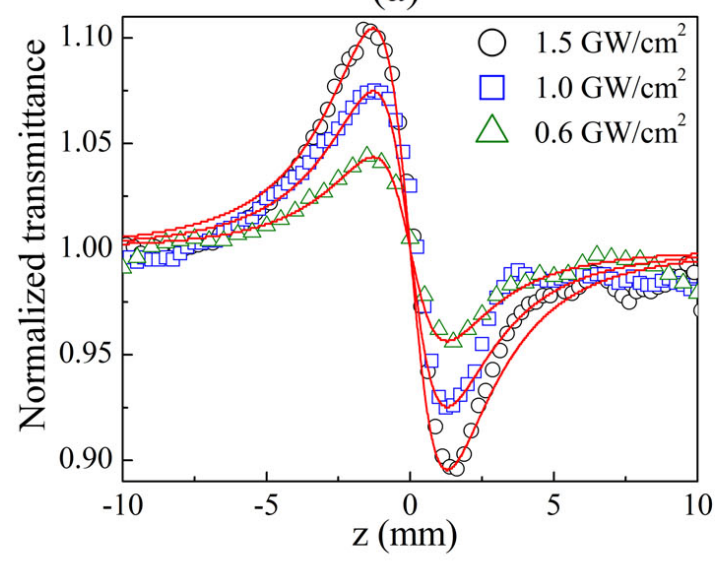

(b)

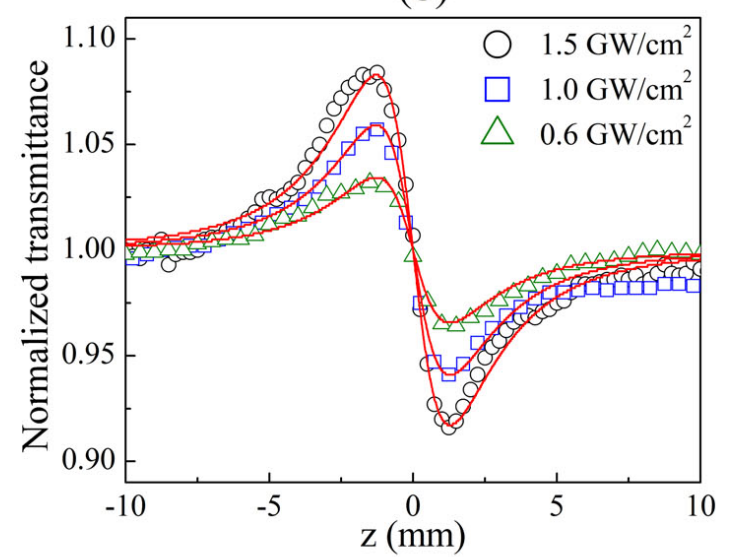

(c)

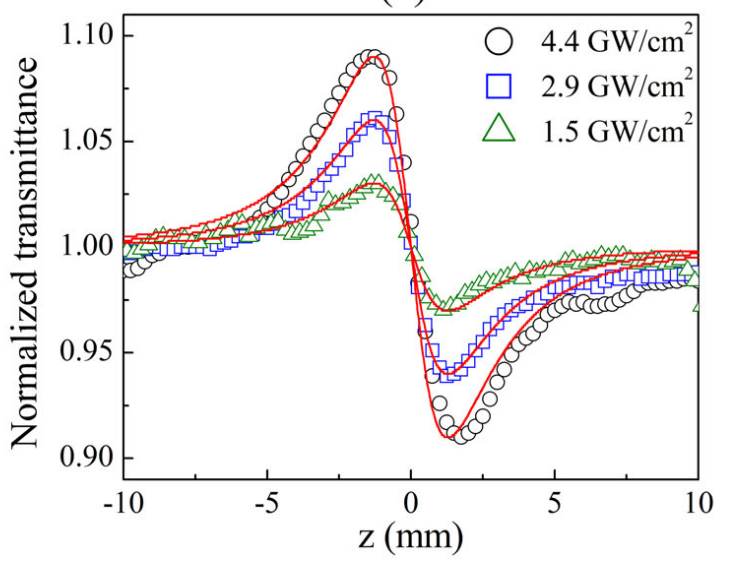

Figure 4(a-c): $\quad$ Normalized transmittance obtained in the CA Z-scan measurement for different incident laser intensities and different concentrations, (a) $10^{-3} \mathrm{M}$, (b) $6.6 \times 10^{-4} \mathrm{M}$ and (c) $10^{-4} \mathrm{M}$. Note the difference in intensities used for the lowest concentration (c).

The experimental values for the nonlinear refraction are obviously dependent on the nanoclusters concentration of the solutions. Hence, it is possible to draw from Figure 4 the $n_{2}$ value as a function of the sample concentration, see Figure 5. Extrapolation to zero concentration shows a third-order refractive index close to zero, because the signal for pure water is smaller than the detection limit. ${ }^{29}$ 


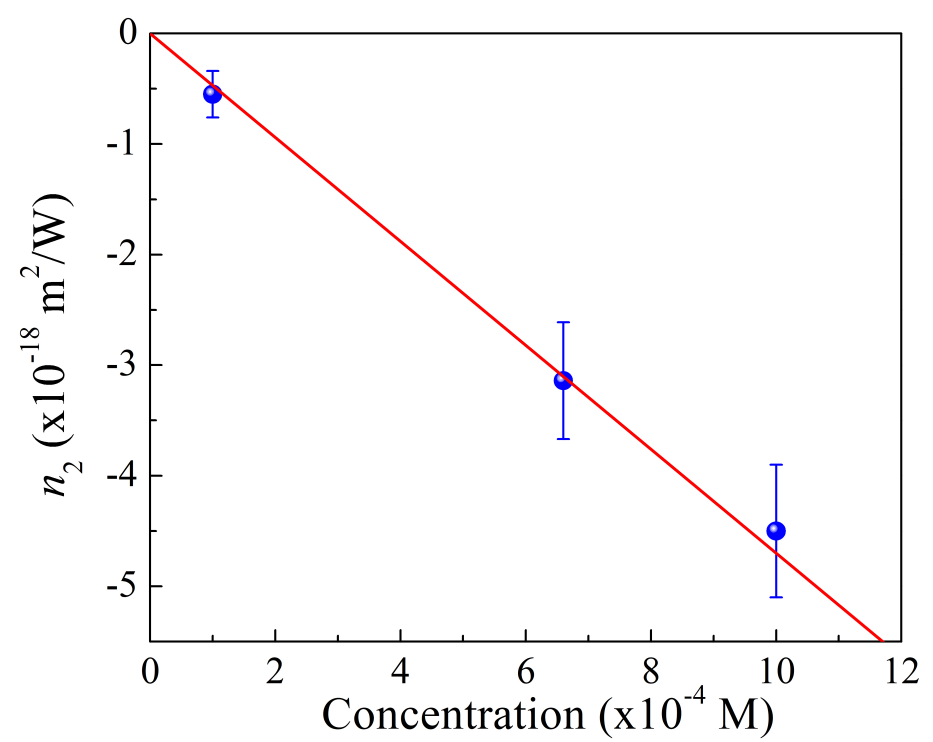

Figure 5: Nonlinear refraction $n_{2}$ obtained from the CA Z-scan measurement as a function of the nanoclusters aqueous solutions concentration.

Following Balu et al., ${ }^{24}$ it is possible to express $n_{2}$ in units of GM, or refractive GM (RGM) to account for the units of $n_{2}$ in $\mathrm{m}^{2} / \mathrm{W}$, using $n_{2}=\lambda N \sigma_{R, \lambda}$ where $\lambda$ is the incident wavelength and $N$ the nanoclusters concentration. Note that in this case the unit of Watts needs to be converted into photons per second using the energy per photon at $532 \mathrm{~nm}$. Then from the slope of Figure 5, we get $\sigma_{R, 532}=(-5.35 \pm 0.5) \times 10^{4} \mathrm{GM}$, a value similar to that of gold nanoclusters, also negative. ${ }^{21}$ Over the whole range of concentration and intensities investigated, the expected linear behavior of $n_{2}$ with concentration is obeyed.

The open-aperture OA Z-scan technique has also been pioneered by Sheik-Bahae et al. ${ }^{28}$ and has been extensively used for evaluating the nonlinear absorption properties of various materials as a very efficient technique. The analysis of the OA Z-scan data are based on a theory of a saturable 2PA using a spatial and temporal Gaussian-profile laser beam. The theory is built on the Adomian decomposition method. ${ }^{30}$ The Z-scan curves were normalized to unity at both distances away from the beam waist. Already at low intensities the 
experimental results exhibit a peak in the transmission curve. This is the signature of the saturation of the 1PA process, see Figure 6 . This is reasonable since the samples are excited at $532 \mathrm{~nm}$, a wavelength slightly longer than that of the longer-wavelength band maximum observed in the absorption spectrum, see Figure 2. Then, with the increase in incident intensity, it is possible to observe the formation of a dip, i.e. also known as a valley, at $z=0$ indicating the appearance of a contribution of a 2PA process, see Figure 6 also. This 2PA process was already reported in previous works and introduced in Eq.(1). ${ }^{21,31}$ Finally, at higher incident intensities, it is possible to observe the saturation of the valley in the Z-scan curves and the formation of a new peak in the region of $z=0$. This re-increase in effective transmission indicates the saturation of the $2 \mathrm{PA}$ process.

(a)

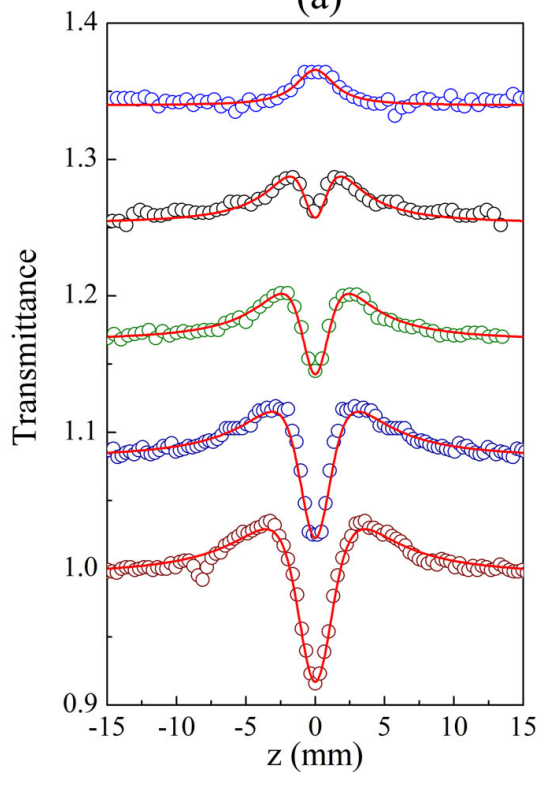

(b)

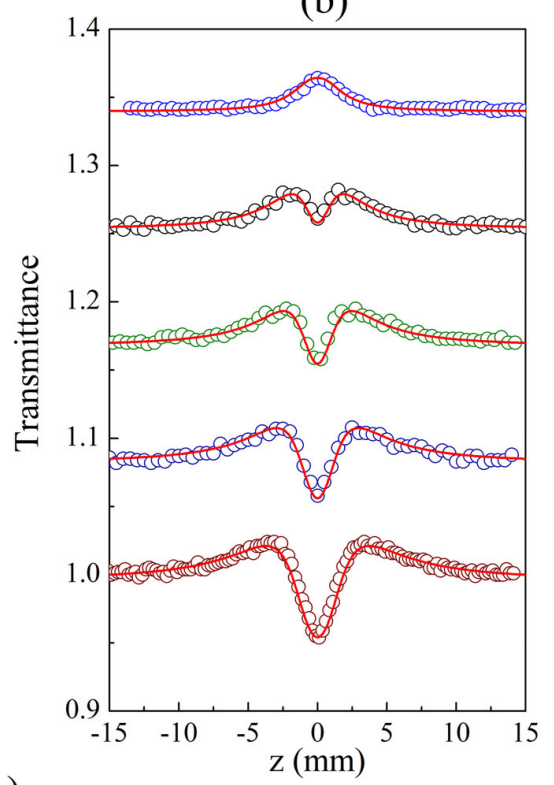

(c)

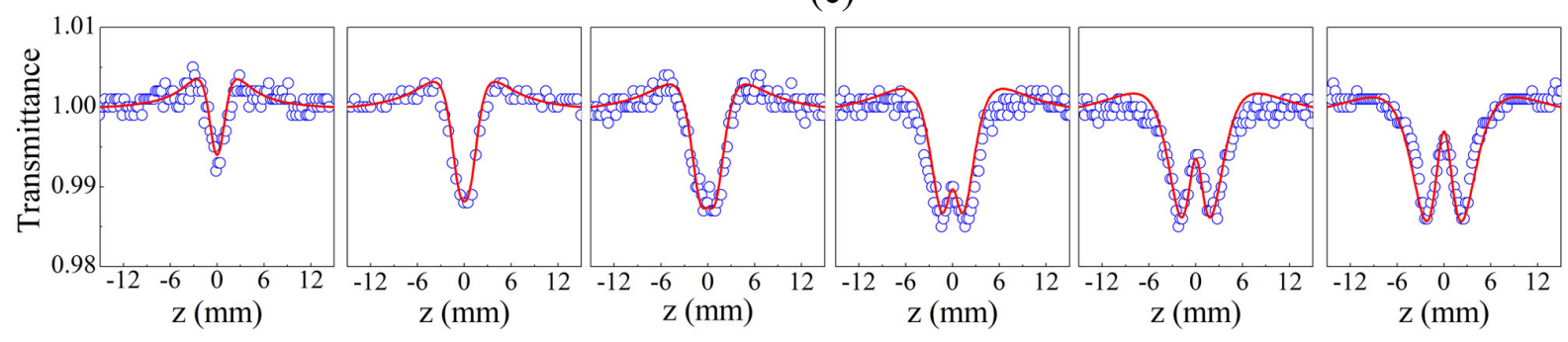

Figure 6(a-c) : Normalized transmittance obtained in the OA Z-scan measurement for different incident laser intensities and different concentrations, (a) $10^{-3} \mathrm{M}$, 
(b) $6.6 \times 10^{-4} \mathrm{M}$ and (c) $10^{-4} \mathrm{M}$. In (a) and (b) the peak intensities from top to bottom correspond to $0.3,1.5,2.2,3.2$ and $4.3 \mathrm{GW} / \mathrm{cm}^{2}$. In (c), from left to right: $1.5,2.9,4.4,7.3,11$ and $15 \mathrm{GW} / \mathrm{cm}^{2}$.

This behavior is clearly observed in the sample with the lowest concentration, i.e. Figure $6 c$, whereas it vanishes at larger concentrations. As the concentration increases, the value of $\alpha_{2}$ increases too and this nonlinear saturation effect is masked. The best agreement for all samples was obtained with the same behavior, i.e. all concentrations were fitted with the same model. In particular, it was not possible to adjust the present data with a nonlinear absorption coefficient of quintic or higher order, notably because several nonlinear contributions would be necessary to model the saturation behavior.

The normalized transmittance due to open-aperture Z-scan was fitted with the following equation :

$$
\frac{d I}{d z}=-\alpha(I) I
$$

where $\alpha(I)$ corresponds to the effective intensity dependent absorption coefficient. However, our results could not be described with the intensity dependent absorption coefficient given in Eq.(1). Addition of the saturation of the nonlinear 2PA process was indeed deemed necessary. This point is further described in the Supplementary Information, see Figure S1. Considering the liquid phase of the sample and the expected inhomogeneous broadening of transitions in these conditions, the saturation of the 1PA and 2PA coefficients take the following expression : 


$$
\alpha(I)=\frac{\alpha_{0}}{\sqrt{1+\left(\frac{I}{I_{\text {sat }, 1 P A}}\right)}}+\frac{\alpha_{2} I}{\sqrt{1+\left(\frac{I}{I_{\text {sat }, 2 P A}}\right)^{2}}} .
$$

The difference of the denominators, compared to Eq.(1) and between the two terms for the powers between $\left(I / I_{s a t, 1 P A}\right)$ and $\left(I / I_{\text {sat }, 2 P A}\right)^{2}$, relates to the models used for the saturation of the 1PA and 2PA coefficients. These models have been discussed previously and are determined by solving the rate equations for a two-level system. ${ }^{32-36}$ Eq.(1) makes use of the homogeneous model for a two-level system. Although rather simple, it lacks the inhomogeneous broadening of resonances encountered in liquid solutions introduced in Eq.(5) for both the linear 1PA and nonlinear 2PA absorption.

Further analysis of the nonlinear absorption as a function of the nanoclusters concentration of the solutions yield linear increasing plots, see Figure 7. Figure 7a is corrected for the absorption of the cell in absence of nanoclusters. This is the standard feature expected for nonlinear absorption. Indeed, we expect the following relationship $\alpha_{2}=\sigma_{\lambda} N$ where $\sigma_{\lambda}$ is the 2PA cross-section per nanocluster at wavelength $\lambda$ and $N$ the nanoclusters concentration. Numerically, we determined $\sigma_{532}=(5.2 \pm 0.5) \times 10^{5} \mathrm{GM}$ at $532 \mathrm{~nm}$ from the slope of the linear plot in Figure 7a. This value has to be compared with the value previously obtained at $800 \mathrm{~nm}$, namely $\sigma_{800}=12930 \mathrm{GM}$. The ratio $\sigma_{532} / \sigma_{800} \approx 40$ is here unambiguously attributed to the possibility to have sequential 1PA processes besides a resonant 2PA process since at $532 \mathrm{~nm}$, linear absorption does not vanish, likewise at $266 \mathrm{~nm}$, the wavelength corresponding to the direct absorption of two photons, whereas it does at $800 \mathrm{~nm}$. In the latter long wavelength case, only the $2 \mathrm{PA}$ process is present and resonant. The two saturation intensities, for the linear and nonlinear regime are however independent of the nanoclusters concentration. 
(a)

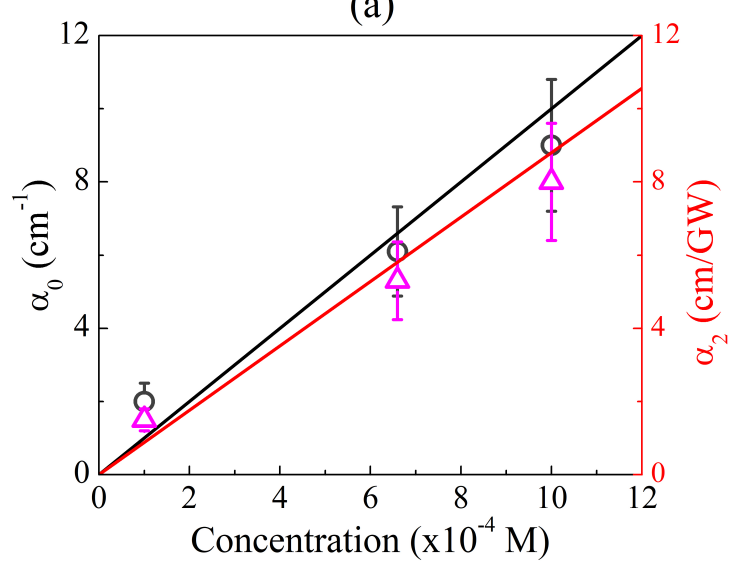

(b)

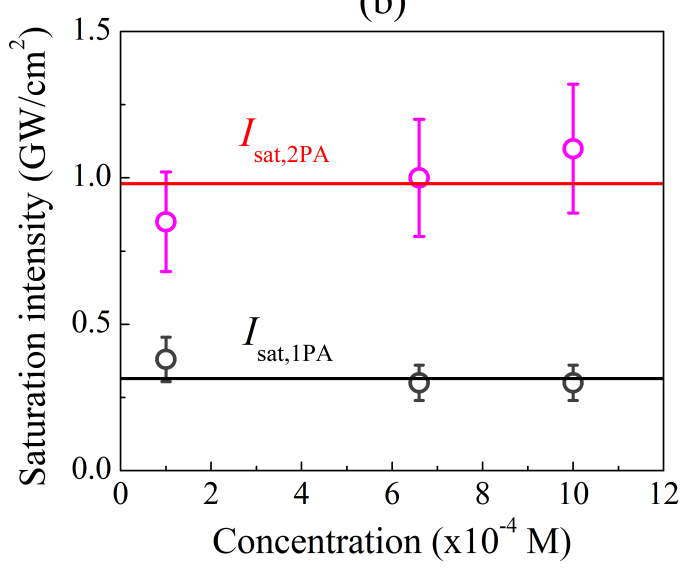

Figure 7: Concentration dependence of the (a) 1PA and 2PA coefficients as well as the (b) linear and nonlinear saturation intensities.

In line with an expected constant value with respect to incident intensity, the saturation intensity for the $2 \mathrm{PA}$ process is relatively large at about $1 \mathrm{GW} / \mathrm{cm}^{2}$ and constant within experimental error. Then, using the different parameters determined, it is then possible to evaluate the nonlinear absorption as a function of the incident light intensity, see Figure 8 . After an initial drop, there is a large increase at all concentrations followed by a decrease afterwards with a shift of the absorption maximum with concentration to larger incident intensities. The main difference lies in the decrease of absorption at large intensities, which is much flattened for high concentrations. 
Figure 8: Incident intensity behavior of the total absorption for three concentrations of the nanoclusters solutions used.

Finally, it is interesting to compare the figure-of-merit defined as $F O M_{\lambda}=\sigma_{R, \lambda} / \sigma_{\lambda}$. This ratio determines the potential of the nonlinear optical material for applications like optical switching where large $n_{2}$ values are required. However, especially for resonance enhanced systems, a weak $2 \mathrm{PA}$ absorption must be preserved. This figure of merit is about $F O M_{800} \sim 1$ for efficient organic compounds ${ }^{24}$ whereas for the present silver nanoclusters in aqueous solutions, we obtain already FOM $M_{532} \sim 0.1$ without specific nanoclusters engineering. This $F O M_{\lambda}$, although smaller than what is observed for other materials like organic materials, ${ }^{24}$ constitutes a starting point to develop nanoclusters based materials for optical switching applications with competitive $F O M_{\lambda}$ since it shows that nonlinear refractive index effects is not fully destroyed by a simultaneous nonlinear absorption. 


\section{Conclusions}

We have reported the nonlinear refraction and absorption coefficients for aqueous solutions of the $\mathrm{Ag}_{29} \mathrm{DHLA}_{12}$ nanoclusters using OA and CA Z-scan experiments. As opposed to previous works, we observe that the linear 1PA and nonlinear 2PA absorption coefficients are both saturated. We emphasize that saturation of the nonlinear absorption was not previously reported for metal clusters. A balance between high incident intensity and low concentration is nevertheless required to clearly observe this feature. High concentrations can indeed mask the saturation of the nonlinear absorption.

The present observation will further help in the understanding of the nonlinear spectroscopy of nanoclusters and the design of optical properties targeted for specific applications. ${ }^{37}$ As one possibility, it is shown using a simple figure of merit that optical switching can be targeted although further engineering on the nanoclusters and their containing matrix must be developed.

It is worth highlighting that although the nonlinear optical properties of metal nanoparticles continue to be deeply explored up to now, they suffer a great limitation in applications that require high efficiency due to their large intensity losses induced by linear and nonlinear absorption effects. ${ }^{38}$ In this way, the observation of the saturation of 1PA and 2PA cross-sections, the last one being shown here for the first time, reveal the metal nanoclusters as a possible alternative to continue exploring the large nonlinear refractive behavior, characteristic of metal particles in nano and sub-nanometer dimensions, by managing the intensity losses through the saturated-absorption regions. Thus, effects such as the nonlinear light pulse propagation over long distances as well as the efficiency of alloptical switching can be benefited by using metal nanoclusters instead of metal nanoparticles.

\section{Supporting Information}


Material supplied as Supporting Information : 1) Description of the different available adjustment models, 2) Parameters of the adjustment models.

\section{Acknowledgement}

The authors acknowledge the support from the CAPES-COFECUB program between Lyon and Recife under contract Sv852-15. The Agence National pour la Recherche (ANR) is also acknowledged for the financial support under project ANR-CE24-2017-0029. This work was performed in the framework of the National Institute of Photonics (INFO) project supported by the Conselho Nacional de Desenvolvimento Científico e Tecnológico (CNPq) and the Fundação de Amparo à Ciência e Tecnologia do Estado de Pernambuco (FACEPE).

\section{References}

(1) Ganeev, R. A. Nonlinear Optical Properties of Materials; Springer Series in Optical Sciences, Springer, Dordrecht, 2013

(2) de Araújo, C. B.; Gomes, A. S. L.; Boudebs, G. Techniques for Nonlinear Optical Characterization of Materials: a Review. Rep. Prog. Phys. 2016, 79, 036401.

(3) Christodoulides, D. N.; Khoo, I. C.; Salamo, G. J.; Stegeman, G. I.; Van Stryland, E. W. Nonlinear Refraction and Absorption: Mechanisms and Magnitudes. Adv. Opt. Photon. 2010, 2, 60-200.

(4) Fainman, Y.; Ma, J.; Lee, S. H. Nonlinear Optical Material Applications. Mater. Sci. Rep. 1993, 9, 53-139.

(5) Principles and Applications of Nonlinear Optical Materials. Munn, R. W., Ironside C. N., Eds.; Springer, Dordrecht, 1993 
(6) Springer Handbook of Lasers and Optics, Träger, F., Ed.; Chapter 5, Springer, Berlin, 2007

(7) Min, W.; Freudiger, C. W.; Lu, S.; Xie, X. S. Coherent Nonlinear Optical Imaging: Beyond Fluorescence Microscopy. Ann. Rev. Phys. Chem. 2011, 62, 507-530

(8) Garmire, E. Nonlinear Optics in Daily Life. Opt. Express 2013, 21, 30532-30544

(9) Zhang, Y.-X.; Wang, Y.-H. Nonlinear Optical Properties of Metal Nanoparticles: a Review. RSC Adv. 2017, 7, 45129-45144

(10) Palpant, B. Third-Order Nonlinear Optical Response of Metal Nanoparticles in NonLinear Optical Properties of Matter, Papadopoulos, M. G., Sadlej, A. J., Leszczynski, J., Eds., Springer, Dordrecht, 2006

(11) Reyna, A. S.; de Araújo, C. B. High-Order Optical Nonlinearities in Plasmonic Nanocomposites - a Review, Adv. Opt. Photon. 2017, 9, 720-774

(12) Drachev, V. P.; Kildishev, A. V.; Borneman, J. D.; Chen, K.-P.; Shalaev, V. M.; Yamnitskiy, K.; Norwood, R. A.; Peyghambarian, N., Marder, S. R.; Padilha, L. A. et al. Engineered Nonlinear Materials using Gold Nanoantenna Array. Sci. Rep. 2018, 18, 780

(13) Ray, P. C. Size and Shape Dependent Second Order Nonlinear Optical Properties of Nanomaterials and their Application in Biological and Chemical Sensing. Chem. Rev. $\mathbf{2 0 1 0}, 110,5332-5365$

(14) Stepanov, A. L., Nonlinear Optical Properties of Implanted Metal Nanoparticles in Various Transparent Matrices: A Review. Rev. Adv. Mater. Sci. 2011, 27, 115-145

(15) Philip, R.; Chantharasupawong, P.; Qian, H.; Jin, R.; Thomas, J. Evolution of Nonlinear Optical Properties: From Gold Atomic Clusters to Plasmonic Nanocrystals. Nano Lett. 2012, 12, 4661-4667

(16) Philip, R.; Kumar, G. R.; Sandhyarani, N.; Pradeep, T. Picosecond Optical 
Nonlinearity in Monolayer-Protected Gold, Silver and Gold-Silver Alloy Nanoclusters. Phys. Rev. B 2000, 62, 13160

(17) Ramakrishna, G.; Varnavski, O.; Kim, J.; Lee, D.; Goodson III, Th. Quantum-Sized Gold Clusters as Efficient Two-Photon Absorbers. J. Am. Chem. Soc. 2008, 130, 50325033

(18) Russier-Antoine, I.; Bertorelle, F.; Vojkovic, M.; Rayane, D.; Salmon, E.; Jonin, Ch.; Dugourd, Ph.; Antoine, R.; Brevet, P. F. Nonlinear Optical Properties of Gold Quantum Clusters. The Smaller the Better. Nanoscale 2014, 6, 13572-13578

(19) Knoppe, S.; Vanbel, M.; van Cleuvenbergen, S.; Vanpraet, L.; Bürgi, Th.; Verbiest, Th. Nonlinear Optical Properties of Thiolate-Protected Gold Clusters. J. Phys. Chem. C 2015, 119, 6221-6226

(20) Mai, H. H.; Kaydashev, V. E.; Tikhomirov, V. K.; Janssens, E.; Shestakov, M. V.; Meledina, M.; Turner, S.; Van Tendeloo, G.; Moshchalkov, V. V.; Lievens, P. Nonlinear Optical Properties of Ag Nanoclusters and Nanoparticles Dispersed in a Glass Host. J. Phys. Chem. C 2014, 118, 15995-16002

(21) Olesiak-Banska, J.; Waszkielewicz, M.; Matczyszyn, K.; Samoc, M. A Closer Look at Two-Photon Absorption, Absorption Saturation and Nonlinear Refraction in Gold Nanoclusters. RSC Adv. 2016, 6, 98748-98752

(22) Sridharanet, K.; Sreekhant, P.; Park, T. J.; Philip, R. Nonlinear Optical Investigations in Nine-Atom Silver Quantum Clusters and Graphitic Carbon Nitride Nanosheets. $J$. Phys. Chem. C 2015, 119, 16314-16320

(23) Russier-Antoine, I.; Bertorelle, F.; Hamouda, R.; Rayane, D.; Dugourd, Ph.; Sanader, Ž.; Bonačić-Koutecký, V.; Brevet, P. F.; Antoine, R. Tuning Ag29 Nanocluster Light Emission from Red to Blue with One and Two-Photon Excitation. Nanoscale 2016, 8, 2892-2898 
(24) Balu, M.; Padilha, L. A.; Hagan, D. J.; Van Stryland, E. W.; Yao, S.; Belfield, K.; Zheng, S.; Barlow, S.; Marder, S. Broadband Z-scan Characterization using a HighSpectral-Irradiance, High-Quality Supercontinuum. J. Opt. Soc. Am. B 2008, 25, 159165 and J. Opt. Soc. Am. B, 2009, 26, 1663-1663 (erratum).

(25) Adhikari, B.; Banerjee, A. Facile Synthesis of Water-Soluble Fluorescent Silver Nanoclusters and HgII Sensing. Chem. Mater. 2010, 22, 4364-4371

(26) van der Linden, M.; Barendregt, A.; van Bunningen, A. J.; Chin, P. T. K.; ThiesWeesie, D.; de Groot, F. M. F.; Meijerink, A. Characterisation, Degradation and Regeneration of Luminescent Ag29 Clusters in Solution. Nanoscale 2016, 8, 1990119909

(27) Reichert, M.; Hu H.; Ferdinandus, M. R.; Seidel, M.; Zhao, P.; Ensley, T. R.; Peceli, D.; Reed, J. M.; Fishman, D. A.; Webster, S.; Hagan, D. J.; Van Stryland, E. W. Temporal, Spectral and Polarization Dependence of the Nonlinear Optical Response of Carbon Disulfide. Optica, 2014, 1, 436-445

(28) Sheik-Bahae, M.; Said, A. A.; Wei, T. H.; Hagan, D. J.; Van Stryland, E.W. Sensitive Measurement of Optical Nonlinearities using a Single Beam. IEEE J. Quantum Electron. 1990, 26, 760-769

(29) Schnebelin, C.; Cassagne, C.; de Araújo, C. B.; Boudebs, G. Measurements of the Third- and Fifth-Order Optical Nonlinearities of Water at 532 and $1064 \mathrm{~nm}$ using the

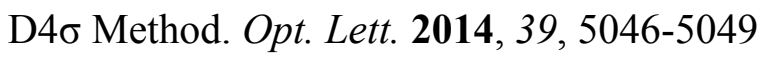

(30) Adomian, G. A review of the decomposition method in applied mathematics. J. Math. Anal. Appl. 1988, 135, 501.

(31) Ferrari, P.; Upadhyay, S.; Shestakov, M. V.; Vanbuel, J.; De Roo, B.; Kuang, Y.; Di Vece, M.; Moshchalkov, V. V.; Locquet, J.-P.; Lievens, P.; Janssens, E. Wavelength- 
Dependent Nonlinear Optical Properties of Ag Nanoparticles Dispersed in a Glass Host. J. Phys. Chem. C 2017, 121, 27580-27589.

(32) Gu, B.; Fan, Y.-X.; Wang, J.; Chen, J.; Ding, J.; Wang, H.-T.; Guo, B. Characterization of Saturable Absorbers using an Open-Aperture Gaussian-Beam $Z$ Scan. Phys. Rev. A 2008, 73, 065803.

(33) Gu, B.; Fan, Y.-X.; Chen, J.; Wang, H.-T.; He, J.; Ji, W. Z-Scan Theory of TwoPhoton Absorption Saturation and Experimental Evidence. J. Appl. Phys. 2007, 102, 083101

(34) Kirkpatrick, S. M.; Naik, R. R.; Stone, M.O. Nonlinear Saturation and Determination of the Two-Photon Absorption Cross-Section of Green Fluorescent Protein. J. Phys. Chem. B 2001, 105, 2867-2873

(35) Yang, L.; Dorsinville, R.; Wang, Q. Z.; Ye, P. X.; Alfano, R. R.; Zamboni, R.; Taliani, C. Excited-State Nonlinearity in Polythiophene Thin Films Investigated by the Z-Scan Technique. Opt. Lett. 1992, 17, 323-325

(36) Swartzlander, Jr. G. A.; Yin, H.; Kaplan, A. E. Continuous-Wave Self-Deflection Effect in Sodium Vapor. J. Opt. Soc. Am. B 1989, 6, 1317-1325

(37) Ho-Wu, R.; Yau, S. H.; Goodson III, Th. Linear and Nonlinear Optical Properties of Monolayer-Protected Gold Nanocluster Films. ACS Nano 2016, 10, 562-572

(38) Khurgin, J. B.; Sun, G. Third-Order Nonlinear Plasmonic Materials: Enhancement and Limitations. Phys. Rev. A 2013, 88, 053838. 


\section{Graphical Abstract}

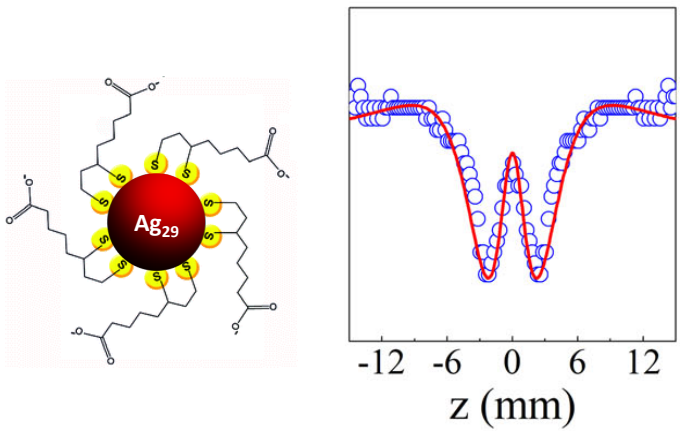

16

14

5

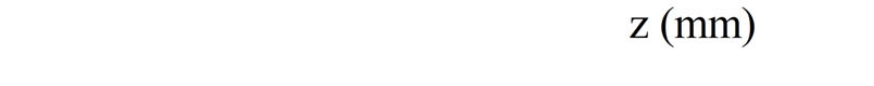

